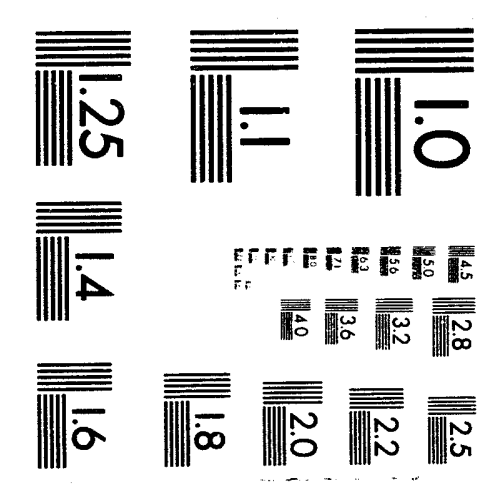




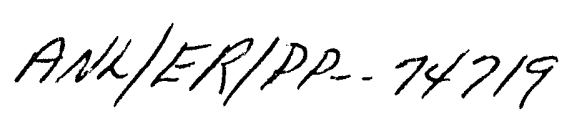

\title{
HIERARCHICAL FRAMEWORK FOR COUPLING A BIOGEOCHEMICAL TRACE GAS MODEL TO A GENERAL CIRCULATION MODEL*
}

\author{
Norman L. Miller \\ Environmental Research Division \\ Argonne National Laboratory, Argonne, IL 60439 \\ and \\ Ian T. Foster \\ Mathematics and Computer Science Division \\ Argonne National Laboratory, Argonne, IL 60439 \\ RECEIVED \\ APR 181994 \\ OSTI
}

Abstract

A scheme is described for the computation of terrestrial biogeochemical trace gas fluxes in the context of a general circulation model. This hiei archical system flux scheme (HSFS) incorporates five major components: (i) a general circulation nodel (GCM), which provides a medium-resolution (i.e., $1^{\circ}$ by $1^{\circ}$ ) simulation of the atmospheric circulation; (2) a procedure for identifying regions of defined homogeneity of surface type within GCM grid cells; (3) a set of surface process models, to be run within each homogeneous region, which include a biophysical model, the Biosphere Atmospheric Transfer Scheme (BATS), and a biogeochemical model (BGCM); (4) an interpolation/integration system that transfers information between the GCM and surface process models with finer resolution; and (5) an interactive data array based on a geographic information system (GIS), which provides land characteristic information via the interpolator. The goals of this detailed investigation are to compute the local and global sensitivities of trace gas fluxes to GCM and BATS variables, the effects of trace gas fluxes on global climate, and the effects of global climate on specific biomes.

*Work supported by the U.S. Department of Energy, Office of Energy Research, Office of Health and Environmental Research, under contract W-31-109-Eng-38. 


\section{INTRODUCTION}

Atmospheric trace gases that absorb thermal infrared radiation add heat to the troposphere. Although carbon dioxide is well known as a significant greenhouse gas (Keeling et al., 1976), other less prevalent trace gases (methane, ozone, nitrous oxide, nitrogen oxides, chloroflorocarbons) are being recognized as having significant effects on global climate (Bolin et al., 1986; Ramanathan, 1975). Dickinson and Cicerone (1987) infer that further additions of these trace gases at current rates during the next 60 years could double or even quadruple present effects, causing the global average temperature to rise at least $1^{\circ} \mathrm{C}$ and perhaps more than $5^{\circ} \mathrm{C}$.

The flux of trace gases between the earth's surface/subsurface and the lower troposphere represents a major pathway by which greenhouse gases are transported into and out of the atmosphere. A quantitative understanding of terrestrial trace gas storages, sources, sinks, and transfers will significantly add to our understanding of global change.

The net flux of biogeochemical trace gases between the terrestrial ecosphere and the lower atmosphere is a significant source of greenhouse gases that has not been modeled on a global scale in the context of a general circulation model (GCM). Such an undertaking requires a detailed representation of numerous interdependent processes that take place on several temporal and spatial scales (Matson, 1990). To approach a quantifiable simulation, a computable general framework that bridges the scales of interaction must be defined. Spatial scales range from the global scale with grid point spacing up to hundreds of kilometers, down to the biome scale where homogeneous areas may be less than a square kilometer. Temporal scales range from minutes up to decades and centuries. A modeling system that simulates the multidirectional flow of trace gases between biome scale biogeochemical processes and global scale atmospheric phenomena is termed here a hierarchical systems flux scheme (HSFS).

An HSFS consists of the five major components illustrated in Fig. 1:

1. A GCM, which provides a medium-resolution (i.e., $1^{\circ}$ by $1^{\circ}$ ) simulation of the atmospheric circulation.

2. A procedure for identifying regions of defined homogeneity of surface type within GCM grid cells the HTEST. 
3. A set of surface process models to be run within homogeneous regions. These include a biophysical model, the Biosphere Atmospheric Transfer Model (BATS) (Dickinson et al., 1986)] and a biogeochemical model (BGCM) for specific biomes.

4. An interpolation/integration system that transfers information between the GCM and with surface process models finer-resolution.

5. An interactive data array based on a geographic information system (GIS), which provides land characteristic information via the interpolator.

A variety of quantitative functions describing trace gas transfer can be linked into this modeling framework (as part of the BGCM) in a modular fashion. Initially, it is intended to use simple parameterizations of net fluxes for each characteristic surface type. Subsequently, it may prove useful to incorporate more detailed numerical models of undivided biomes. Incorporation of more detailed biogeochemical models and functions provides a basis for computing the effects of climatic changes on individual biomes. For example, effects due to global heating can be incorporated into the surface biophysical and biogeochemical processes.

The level of detail to be provided by this modeling framework leads to large computational requirements. This issue will be addressed by the use of high-performance parallel computers. In particular, the BGCM and other system components will be integrated with a parallel implementation of the Community Climate Model (CCM-2) being developed at Argonne National Laboratory, Oak Ridge National Laboratury, and the National Center for Atmospheric Research as (NCAR) part of the Department of Energy's CHAMMP program (Foster et al, 1991, Drake et al., 1991). However, the modeling framework defined here is designed to be portable to other GCMs.

The rest of this paper describes the five components in the hierarchical system framework in detail. It also outlines the studies that will be performed to test and validate the modeling system.

\section{DISCLAIMER}

This report was prepared as an account of work sponsored by an agency of the United States Government. Neither the United States Gnvernment nor any agency thereof, nor any of their employees, makes any warranty, express or implied, or assumes any legal liability or responsibility for the accuracy, completeness, or usefulness of any information, apparatus, product, or process disclosed, or represents that its use would not infringe privately owned rights. Reference herein to any specific commercial product, process, or service by trade name, trademark, manufacturer, or otherwise does not necessarily constitute or imply its endorsement, recommendation, or favoring by the United States Government or any agency thereof. The views and opinions of authors expressed herein do not necessarily state or reflect those of the United States Government or any agency thereof. 


\section{FRAMEWORK FOR BIOGEOCHEMICAL FLUX CALCULATION}

\section{A. General Circulation Model}

A GCM resides at the top level of the computational pyramid of the HSFS. This component solves the equations of motion, temperature, density, hydrostatics, and state to yield horizontal (sigma surface) fluid dynamic fields on a sphere. Atmospheric physics of clouds, radiation, etc. are computed as vertical columns at single horizontal grid point locations. A GCM to be used for computing trace gas fluxes must be able to transport a variety of trace gases with nonuniform distributions. The most recent version of the Community Climate Model (CCM2) provides this capability.

The GCM component of the HSFS provides the surface process models (discussed below) with information about temperature, precipitation, winds, etc. It receives data on the biophysical and biogeochemical fluxes from surface process models.

\section{B. HTEST: Identification of Homogeneous Regions}

The hierarchical systems flux model uses a procedure called HTEST to identify subregions of defined homogeneity within a GCM grid cell. The procedure is applied once at the beginning of a simulation. Homogeneity is defined in terms of percentage of uniformity in vegetational and topographic characteristic types. The degree of uniformity required within a subregion will depend on accuracy requirements, available computational resources, and observed variability in flux rates between different surface types.

HTEST identifies homogeneous regions within a GCM grid cell by first applying a homogeneity test to the entire cell. If the test fails, the cell is divided in two, and the test is repeated in each nonhomogeneous subcell. This bisection process continues (alternating northsouth and east-west divisions) until either all subcells have been characterized as homogeneous or a defined minimum resolution has been reached. (For example, if a GCM grid cell has size $1^{\circ}$ by $1^{\circ}$, then ten bisections would yield approximately $3-\mathrm{km} \times 3-\mathrm{km}$ resolution). Figure 2 provides a top view of nine GCM cells and shows the telescoping structure for determining homogeneous land surfaces. Figure 3 represents a single GCM cell with a heterogeneous land surface. Four land types are shown from upper left (clockwise) as types 1,2,3, and 4 for simplicity. The HTEST takes the ratio of a characteristic area to the total area (e.g., HTEST $=($ area $1 /$ areaT $)=0.5)$. 
If this ratio is below a specified value (0.95), then homogeneity has not been artained, and a bisection of the area is computed. Alternating east-west and north-south divisions are illustrated for five bisections.

Application of the HTEST procedure is intended to generate a sparsely refined or telescoping grid in which most GCM cells ( $>90 \%$ ) are not partitioned. Because telescoping occurs in regions where flux gradients are high, this approach should permit a significant improvement in the accuracy of simulation of surface fluxes without greatly increasing computational costs.

In general, the number of bisections is limited by the resolution of the GIS data. For example, a $0.25^{\circ}$ by $0.25^{\circ}$ surface resolution will allow for only two bisections on a global $1^{\circ}$ by $1^{\circ}$ resolution. However, identification of specific regions with a high degree of heterogeneity may be provided with resolution well below $0.1^{\circ}$ by $0.1^{\circ}$. R. Harris has suggested that data for these specific regions are available and will be useful (personal communication, 1991).

This basic framework to identify homogeneous regions will probably have to be extended to deal with point sources of specific trace gases (e.g., bogs). This extension can be achieved by invoking pattern recognition techniques to search for specific characteristic properties in the geographic databases.

\section{Surface Process Models}

Surface process models are applied within each homogeneous region identified by HTEST. These models compute biophysical and biogeochemical processes as a function of the GIS characteristic type and the scaled GCM state variables provided by the interpolation system. The surface process model component comprises two distinct subcomponents: BATS and BGCM. BATS is used to compute surface albedo, roughness, leaf and stem area indices, etc., as a function of characteristic type and scaled GCM state variables. BATS can be run at a wide range of resolutions and is hence well suited for integration in the HSFS. The BGCM computes biogeochemical trace gas fluxes as a function of GIS characteristic type, BATS biophysical variables, and scaled GCM state variables.

The BGCM defines a framework into which parameterizations and system models representing information at the level of biogeochemical processes can be fitted as modules. 
Existing numerical models such as CENTURY (Schimel et al., 1990), ELM (Cole, 1976), and FLEX (White and Overton, 1977) compute detailed ecological processes for time periods ranging from days to centuries. These models need to be modified to compute net trace gas fluxes before they can be efficiently integrated into the present framework. Parameterizations of biogeochemical processes -- quantitative functions based on system models and field observations --are typically less accurate than detailed models but are less expensive computationally.

Initial studies with the HSFS will use simple parameterizations, obtained from the literature, to represent biogeochemical processes (initially methane) for the principal characteristic surface types. In subsequent studies, more detailed models will be introduced. This work will require extensive interaction with ecologists, particularly when process models and parameterizations are verified in conditions simulated by a GCM. The modular nature of this framework makes it straightforward to substitute alternative representations of specific biomes. In particular, faster and more sophisticated trace gas process functions can be incorporated as they become available.

The computed biogeochemical fluxes (net rates at which decomposed carbon and niirogen diffuse from the land to the atmosphere) are passed directly to BATS and are carried with the biophysical fluxes (heat, momentum, moisture) back to the GCM to complete a single grid point computation.

\section{Interpolation/Integration System}

Interpolation functions transfer GCM state variables to the homogeneous regions identified by HTEST. Initially, these functions will be based on those used by Dickinson et al. (1989) for the transfer of GCM state variables to the mesoscale meteorology version 4 (MM4). These functions combine GCM state variables and mesoscale topography to obtain significantly more accurate distributions of precipitation, temperature, etc. Integration functions are required to transfer BGCM trace gas fluxes to the GCM. Since flux per unit area is computed, an unweighted summation over the surface area will be used.

\section{E. Interactive Data: Geographic Information System}

An interactive data array will be used to determine homogeneity and to pass surface information to BATS and BGCM. The vegetative classifications given by Matthews (1983) 
provide one global set of characteristic types. Other critera (e.g., topography, soils, and geology) can be used to obtain more detailed characterizations. This information is available as a GIS from the National Center for Geographic Information and Analysis (Nyerges, 1991). On a global scale, characterizations based on the additional criteria should provide similar geographic classifications, as the boundaries of natural vegetation zones, soil types, and climate regions are nearly aligned (Trewartha, 1968).

The characteristic land data will be formatted as a GIS array with coded values representing each variable. This information will be used by the BGCM for process calculations. In long term simulations, the land characteristic data may need to be periodically updated with newly computed values. This updating could trigger reapplication of the HTEST procedure.

\section{TESTING AND VALIDATION}

An essential first-step in testing the effect of adding computed trace gas fluxes to a GCM is a sensitivity study. Such a study would incorporate maximum flux estimates from the literature and would run a series of flux/no flux scenarios to determine the degree of change in global climate variables. If a significant signal is determined, further tests will be performed to find the minimum flux necessary to maintain a signal. Local studies for regions with high flux values will be examined similarly.

If the representation of trace gas fluxes influences climate prediction, the next stage will be to develop process modules for specific biomes to provide a more accurate representation of trace gas fluxes. An important aspect of this work will be the validation of individual process modules. Each process module will be extensively tested with observational data (when available) and extrapolated to a wide variety of conditions that each process may experience. This test for robustness will reduce the errors due to extending a function beyond its solution domain. Sensitivity tests will be performed to determine the level of response in each parameterized process for a variation in every biophysical and GCM state variable. Less significant processes will be treated more coarsely and perhaps less frequently than the more sensitive processes.

A single column GCM will be used to test the sensitivity to the maximum and minimum flux values for each process. Comparison of process functions and models with long term data studies and with other models will be part of the ongoing contribution of ecologists to this effort. 
The HSFS will be tested as a climate model and compared to its GCM (without trace gas fluxes) and to other existing GCMs. This is the most important test of significance; it will quantify the effect of terrestrial trace gas fluxes.

\section{SUMMARY AND CONCLUSIONS}

The HSFS framework presented in this paper will couple a GCM with subgrid biogeochemical fluxes for a more realistic calculation of the effect of greenhouse gases and climate change. An innovative telescoping grid technique and an interpolation scheme allow for representation of fine details at minimal computational cost. These tools can be adapted for other geophysical modeling problems.

Several components of the HSFS are already in place. The NCAR GCM-Community Climate Model (Williamson et al., 1987) is running on parallel computers. A number of interpolation functions have been defined, coded, and tested. The BATS-BGCM interface has been defined, coded, and tested with a simple BGCM parameterization. A functional representation of carbon fluxes from one biome, the tallgrass prairie, has been partially constructed.

The next step is to integrate BATS-BGCM and the GIS into the GCM and then perform preliminary sensitivity studies for non-telescoping grids. Concurrently with this activity, parameterizations will be developed for a larger number of biomes.

Climatic sensitivity to terrestrial trace gases is a significant and complicated piece in the climate change puzzle. The HSFS framework provides a means of quantifying this sensitivity. The framework can also be used to predict biome change as a function of climate change. However, this step requires more detailed biogeochemical and ecological process information and thus detailed interaction with the ecology community.

\section{REFERENCES}

Bolin, B., B.R. Doos, J. Jager, and R.A. Warrick. 1986. The greenhouse effect. A synthesis of the present knowledge. J. Wiley Publishers. 
Cole, G.W. 1976. ELM: Version 2,0. Range Science Department Science Series No.20, Colorado State University Publishers.

Dickinson, R.E., R.M. Errico, F. Giorgi, and G.T. Bates. 1986. A regional climate model for the western United States. Climatic Change. 15, 383-422.

Dickinson, R.E. and R.J. Cicerone. 1986. Future global warming from atmospheric trace gases. Nature, 319, 109-115.

Drake, J., I. Foster, P. Swarbauber. 1991. Progress report of the CHAMMP interorganizational team for numerical simulation, Technical Report, ANL.

Foster, I.T., W. Gropp, and R. Stevens. 1991. The parallel scalability of the spectral transform method, Mon. Wea. Rev. (in press).

Keeling, C.D., R. B. Bacastow, A. E. Bainbridge, C.A. Ekdahl Jr., P.R. Guenther, and L.S. Waterman. 1976. Atmospheric carbon dioxide variations at Mauna Loa Observeratory, Hawaii. Tellus, 28, 528-551.

Matson, P.H. 1990. Statistical analysis of Ecological response to large-scale perturbations, Ecological Society of Amer.

Matthews, E. 1983. Global vegetation and land use: New high resolution data bases for climate studies. J. Clim. Appl. Meteor., 22, 474-487.

Nyerges, T.L. 1991. GIS for environmental modelers: An overview, First Int. Conf./Workshop on Integrating GIS and Env. Mod., Sept. 19-19, 1991, Boulder, Colorado.

Ramanathan, V. 1975. Greenhouse effect due to chloroflourocarbons: Climatic implications, Science, 190, 50-52.

Schimel, D.S., T.G.F. Kittel, and W.J. Parton, 1990. Terrestrial biogeochemical cycles: Global interactions with the atmosphere and hydrology (submitted to Tellus).

Trewartha, G.T. 1968. An introduction to climate. McGraw Hill, New York.

White, C. and S. Overton. 1977. Users manual for the FLEX2 and FLEX3: Model processors for the FLEX modelling paradigm. School of Forestry, Oregon State University Publishers.

Williamson, D.L., J.T. Kiehl, R.E. Dickinson, and J.J. Hack. 1987. Description of the NCAR community climate model. NCAR technical note, NCAR/TN-285+STR, National Center for Atmospheric Research, Boulder, Colorado. 


\section{Figure Captions}

Figure 1. The hierarchical systems flux scheme consists of five major components: (1) a general circulation model (GCM), which provides a medium-resolution (e.g., $1^{\circ}$ by $1^{\circ}$ ) simulation of the atmospheric circulation; (2) a procedure for identifying regions of defined homogeneity of surface type within GCM grid cells; (3) a set of surface process models, to be run within each homogeneous region, which will include a biophysical model (e.g., the Biosphere Atunospheric Transfer Model -- BATS) and a biogeochemical model (BGCM); (4) an interpolation/integration system that transfers information between the GCM and surface process models with finer resolution; and (5) an interactive data array based on a geographic information system (GIS), which transports land characteristic information with the interpolator.

Figure 2. The top view of the hierarchical scheme for a set of nine GCM grid cells is shown. Each cell is tested for homogeneity. If the test fails, the cell is bisected, and the test is repeated until homogeneity or a predefined minimum resolution is reached.

Figure 3. The hierarchical structure of a single grid cell is shown. The cell fails the homogeneity test and is divided in two. The GCM data are passed through the interpolator to represent the half-scale grid for the two subcells. Testing and bisecting proceeds until homogeneity is reached over the entire cell. Each subcell runs a version of the biophysical flux model (BATS) and the biochemical flux model (BGCM) and sends the respective fluxes to the interpolator to be summed with the results for other subcells and then passed on to the GCM. 


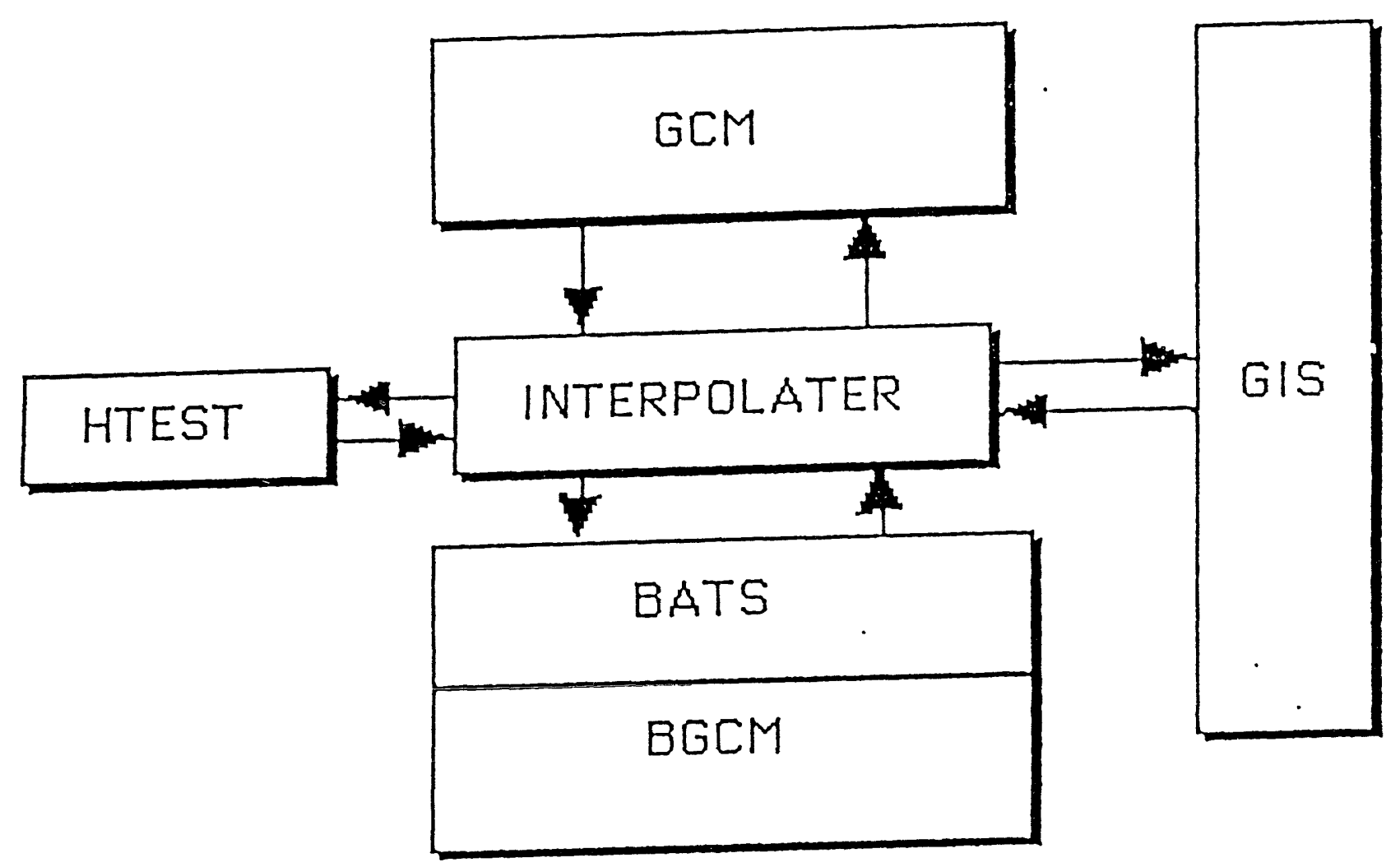

, 


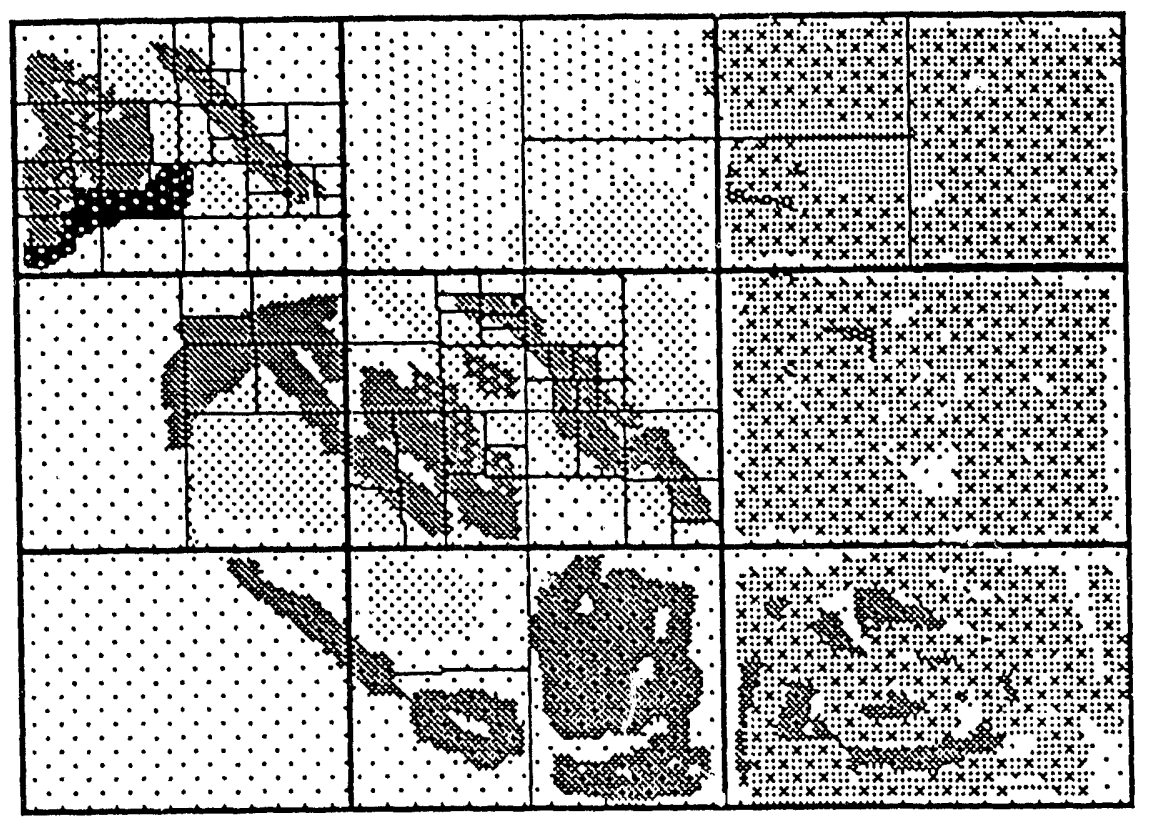




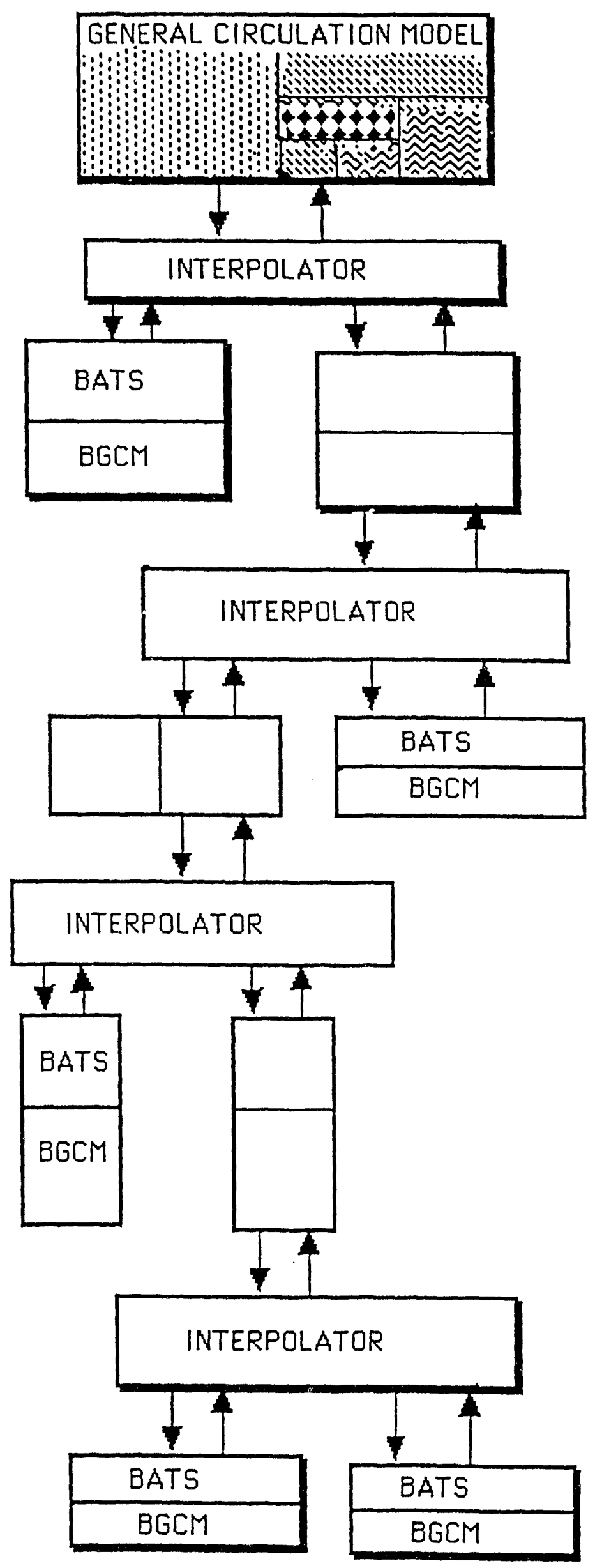


11
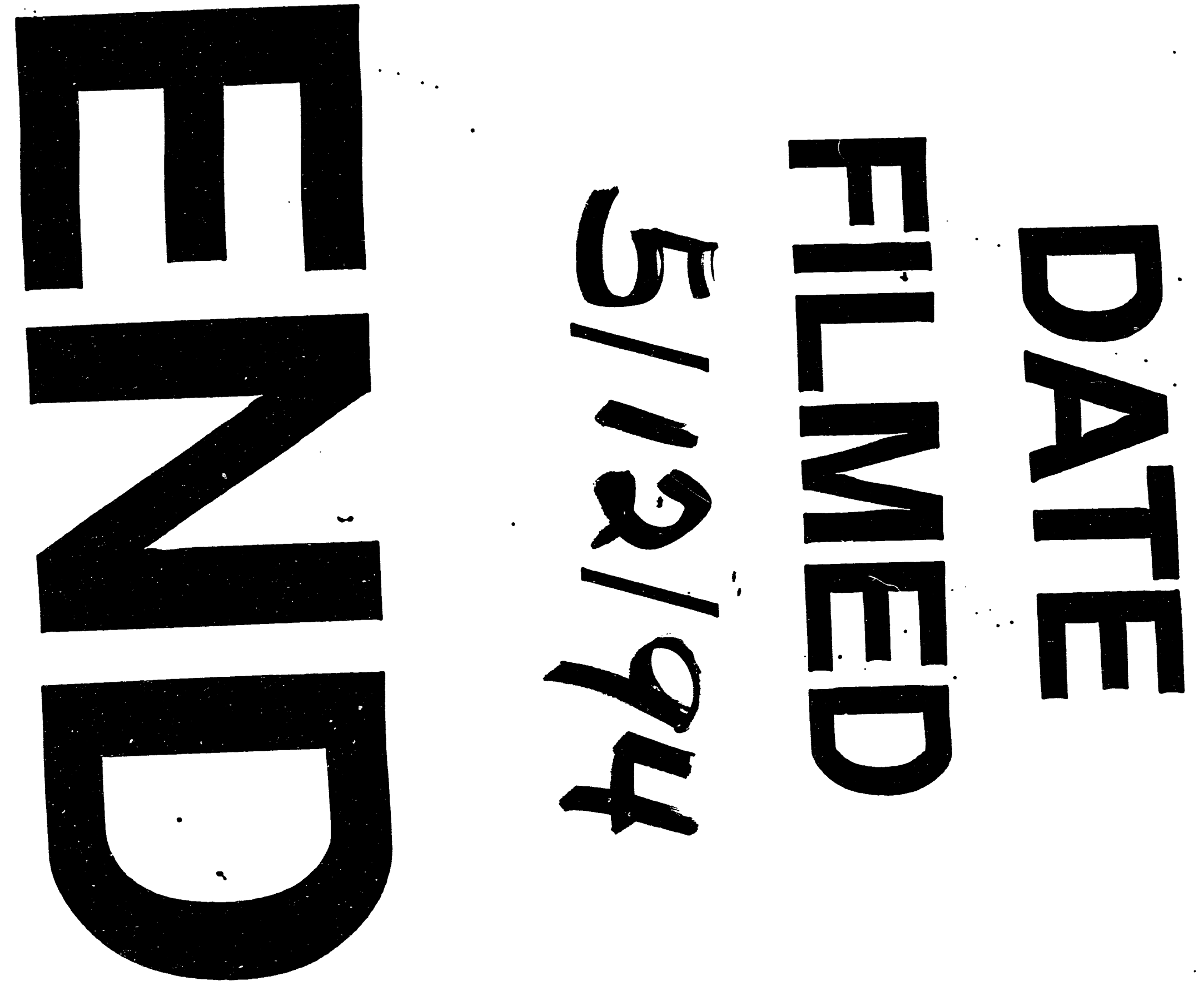
- 